Article

\title{
New Insight into Organic Metal Polyaniline Morphology and Structure
}

\section{Bernhard Wessling}

Ormecon GmbH (a business of Enthone, Inc.), Ferdinand-Harten-Str. 7, D-22949 Ammersbek, Germany; E-Mail: bwessling@ cooksonelectronics.com; Tel.: +49-40-604106-25;

Fax: +49-40-604106-51

Received: 11 October 2010; in revised form: 7 December 2010 / Accepted: 16 December 2010 / Published: 17 December 2010

\begin{abstract}
Polyaniline is known to be a true metal, though a nanometal. Previous experimental and theoretical evidence is reviewed. Two important structural features are presented, which have not publicly been discussed so far: (a) The formation of complexes between polyaniline and metals $(\mathrm{Cu}, \mathrm{Fe}, \mathrm{Zn}, \mathrm{In}$, etc.) which are crucial for most practical applications of the organic metal, polyaniline; and (b) a model for the polyaniline chain structure within the smallest morphological unit, the roughly $10 \mathrm{~nm}$ primary particle.
\end{abstract}

Keywords: polyaniline; organic metal; nanometal; metal complex; morphology; structure

\section{Introduction}

Conductive polymers are principally insoluble and infusible, which has been shown by numerous experimental approaches and by thermodynamical considerations [1]. A "solution" of a conductive polymer would require a single chain having no interaction with a neighbor chain but only interactions with the solvent, i.e., the chain would be completely surrounded by solvent molecules. Such systems do not and cannot exist.

Therefore, they can only be processed by dispersion. "Dispersion" is a process by which (in the case of solids becoming dispersed in a liquid) agglomerated particles are separated from each other and a new interface, between an inner surface of the liquid dispersion medium and the surface of the particles to be dispersed, is generated. Dispersion is a much more complicated (and less well understood) process than most people believe. 
In order to disperse conductive polymers, it must be ascertained what the smallest primary morphological units are that can exhibit all the properties of the macroscopic material. It must also be understood whether these are fibrils, or more or less globular particles? We must know whether they are of micrometer size i.e., 100 nanometers or below?

In a review article [2], all relevant publications (including proprietary work) have been discussed, for example, the discovery of globular primary particles (around $10 \mathrm{~nm}$ in size) which are the primary morphological building unit of all conductive polymers, whether in powder, film or fibrilar form; non-linear phenomena in dispersions (such as rheological properties and sudden conductivity increase at a critical volume concentration); the formation of (so-called) dissipative structures in dispersions; the fact that percolation theory cannot be applied to (polymer matrix based) dispersions of particles having less than $1 \mu \mathrm{m}$ in size; and, finally, the concise non-equilibrium thermodynamic theory for heterogeneous polymer systems, published in two parts $[3,4]$.

Surprisingly, with improved dispersion, not only the conductivity (in contrast to any naive assumption) increased, but also the material crossed the insulator-to-metal boundary to the metallic side [5]. While the transition from an insulator-type of electron transport to a metal-type-happening during dispersion - was surprising, observations that conductive polymers could exhibit metallic properties had already been published before. These various observations, partially also confirmed in our laboratories, had been generalized by the "granular metal concept" [6]. This basically describes the metallic properties in polyaniline, which are understood to be fibrils with more or less straight chains, as emerging in certain limited areas ("islands"), where the chains exhibit a higher degree of order and these "islands" are linked together by less ordered chains, allowing the electrons to move from one "island" to the next.

If this concept is correct, then any dispersion not only would not improve the conductivity, but would in fact deteriorate it (by cutting the chains connecting the "islands"). However, our experiments showed no such deterioration; and on the contrary even finally succeeded in changing from the insulator to the metal side of the IM transition during dispersion. Therefore, the "Organic Nanometal" concept was developed [1] (p. 513), inspired by the experimental and theoretical background on "mesoscopic metals" (i.e., conventional metals like $\mathrm{Cu}, \mathrm{Ag}, \mathrm{In}$, etc. in nanoscopic form) brought forward by G. Nimtz and coworkers [7,8]; which we jointly have been able to show as being very similar to the behavior of polyaniline [9,10]. We found the metallic core with a size of $8 \mathrm{~nm}$ (assuming globular form), within a total porimary particle size of $9.6 \mathrm{~nm}$, is well in accordance with former conclusions from scanning tunneling microscopy (STM) evaluations in which we deducted a particle size of around $10 \mathrm{~nm}$.

The concept outlines in [7] that the transport mechanism is purely metallic within the metallic core while the electrons move to the next particle via a barrier (at least $0.8 \mathrm{~nm}$ for one particle, in total at least $1.6 \mathrm{~nm}$ thick) of a less conductive or even insulating shell around the metallic core, by a mechanism called "tunneling" (sometimes also referred to as "hopping"). Electrons which are tunneling do not need a material type of pathway or file, nor a conductive polymer chain for tunneling through even insulating or empty spaces. As Nimtz has shown later, moreover, electrons do not spend any time within the barrier but arrive at the other side at the same time as they begin tunneling [11].

At the time the "Organic Nanometal Concept" was conceived, we did not know anything about the conductivity values within the metallic core. We had only been able to measure the macroscopic conductivity which is around $5 \mathrm{~S} / \mathrm{cm}$ for the raw polymer powder (in pressed pellet form) at the 
insulator side of the IM transition, and above $100 \mathrm{~S} / \mathrm{cm}$ up to $600 \mathrm{~S} / \mathrm{cm}$ for the metallic polyaniline [12]. Later, in cooperation with two other research groups and using electron spin resonance spectroscopy (EPR), we succeeded to find the intrinsic conductivity within the metallic core to be in the order of 60,000 S/cm [13]. This is at least 100 times more than we can macroscopically measure.

Yet we still did not understand the structural basis for the conductivity, what structural changes occurred during dispersion within the particle, within the metallic core, and which structural pattern is responsible for higher conductivity compared to the lower (and non-metallic) one. There are numerous papers in the scientific literature proposing structures for polyaniline derived from wide-angle x-ray diffraction studies, for example an overview given in [14], including a proposal we have contributed [1] (p. 522), but none have been helpful. The assumption (or "hope") that there would be a correlation between higher conductivity and higher crystallinity (i.e., better resolved wide angle x-ray diffraction pattern) did not eventuate. On the contrary, it appears that the peaks' bandwidths (anyway looking more like spectra of amorphous materials) even get wider with increasing conductivity. The only hint towards a possible mechanism was the finding that the volume of the elementary cell (although the absolute values were, perhaps, not correct) decreases with increasing conductivity. However, at that time, we only investigated a conductivity range over one order of magnitude.

A second indication of what may be happening, and in which direction we should look, came from some first results of a small angle x-ray diffraction (SAXS) [15]. For the first time, we detected a $3.5 \mathrm{~nm}$ small subunit (and confirmed the around $10-15 \mathrm{~nm}$ primary particle size), which we assume to represent the diameter of individual polyaniline chains within the primary particle; the chains not being straight but somehow coiled.

The indication of a volume change along with conductivity increase, and the discovery of a $3.5 \mathrm{~nm}$ subunit raised hope of discovering more insights. A better understanding of the structure/conductivity relationship has quite important significance for the goal of increasing the conductivity of conductive polymers and organic metals by one or two more orders of magnitude, towards the intrinsic conductivity.

For a better understanding, at least of polyaniline, also the existence of its complexes with metals needs to be discerned (such metal complexes are, for instance, not possible for poly-ethylenedioxythiophene, PEDOT). Such complexes have hitertho not been published, except as patents $[16,17]$. Here, we will focus on complexes with ions derived from less noble metals than polyaniline (the polyaniline normal potential is between that of $\mathrm{Cu}$ and $\mathrm{Ag}$ ).

The complex with Ag has been discussed in [18]. This is a complex of a different kind, insofar as it does not involve $\mathrm{Ag}$ ions, but $\mathrm{Ag}$ metal in nanoscopic form. It is used as a very powerful oxidation prevention and solderability preserving nanolayer for copper pads in printed circuit board manufacturing and assembly [19].

In the following, the most recent results will be reported and discussed regarding organic metal/conventional metal complexes, their sub-nanoscopic structure and nanoscopic morphology [20].

\section{Results and Discussion}

\subsection{Polyaniline-Metal complexes}

Polyaniline can form complexes with many metal ions by reacting with the base metal, provided its oxidation potential is lower than polyaniline's potential $[18,19]$. Hence, complexes have been observed forming with $\mathrm{Cu}^{1+}, \mathrm{Fe}^{2+}, \mathrm{Zn}^{2+}, \mathrm{In}^{2+}$ and others. 
When looking too quickly at the reaction of polyaniline with the metal form of $\mathrm{Cu}, \mathrm{Fe}, \mathrm{Zn}$, or $\mathrm{In}$, respectively, it could easily be concluded that a simple oxidation has taken place, and many experimentalists may have concluded this. However, the result is completely different when simply adding $\mathrm{Cu}^{1+}, \mathrm{Fe}^{2+}, \mathrm{Zn}^{2+}$ or $\mathrm{In}^{2+}$ ion salts to a water dispersion of polyaniline, compared to the result when allowing a water dispersion of polyaniline to react with $\mathrm{Cu}, \mathrm{Fe}, \mathrm{Zn}$, or In metal (in granule or powder form), and hence the result of such reaction is much more interesting.

This can easily be seen when comparing the spectra: When mixing the respective metal ions (water solution) with the polyaniline water dispersion, the UV spectra will not change at all, except for some minor dilution effect. However, when following the reaction of polyaniline with the metal (which is a relatively slow reaction taking several hours to complete), the UV spectra gradually change and will ultimately be very different from the starting polyaniline spectrum, as can be seen in Figures 1 to 4 .

Figure 1. UV-Vis spectra for polyaniline and its complexes with $\mathrm{Cu}, \mathrm{Fe}$ and $\mathrm{Zn}$.

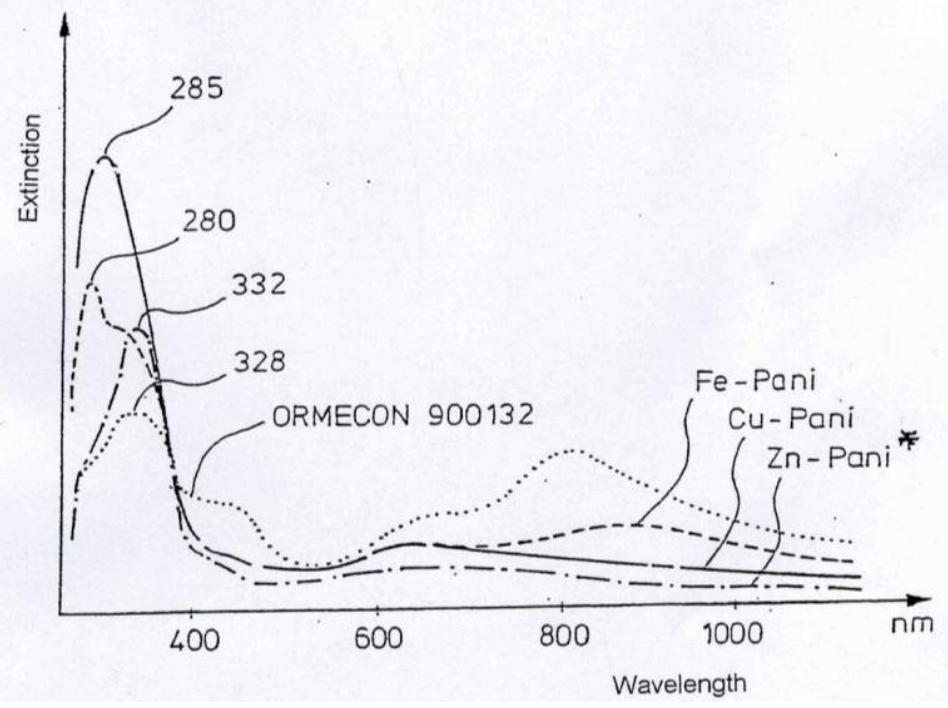

Figure 2. UV-Vis spectrum evolving during the reaction of polyaniline with $\mathrm{Cu}$ metal.

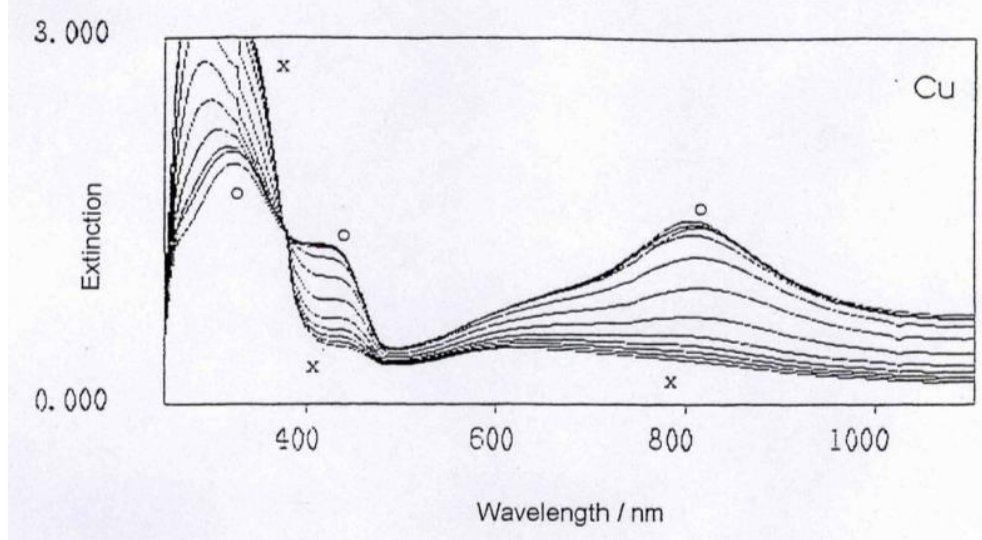


Figure 3. UV-Vis spectrum evolving during the reaction of polyaniline with Fe metal.

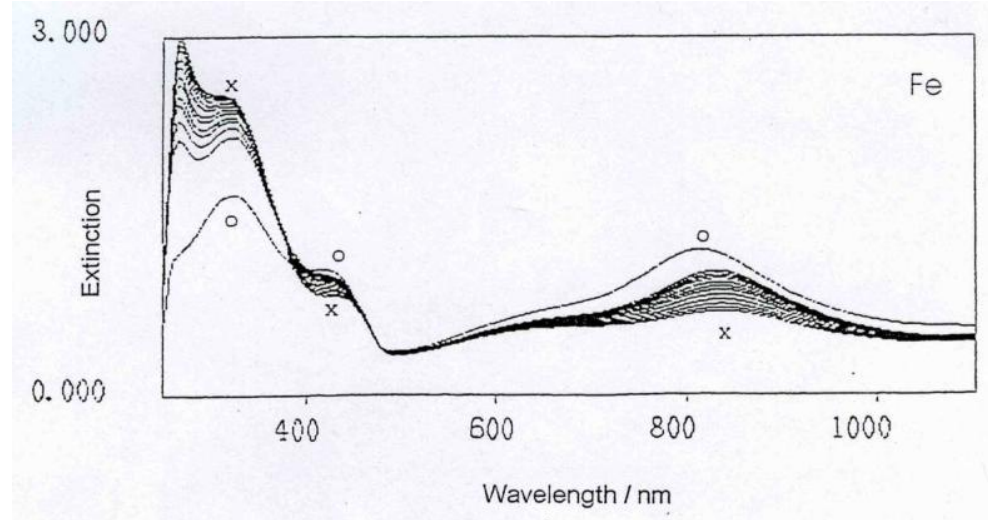

Figure 4. UV-Vis spectrum of polyaniline (pure) and polyanilne/In complex; the curve with the lowest absorption around $800 \mathrm{~nm}$ representing the complex after reaction completed.

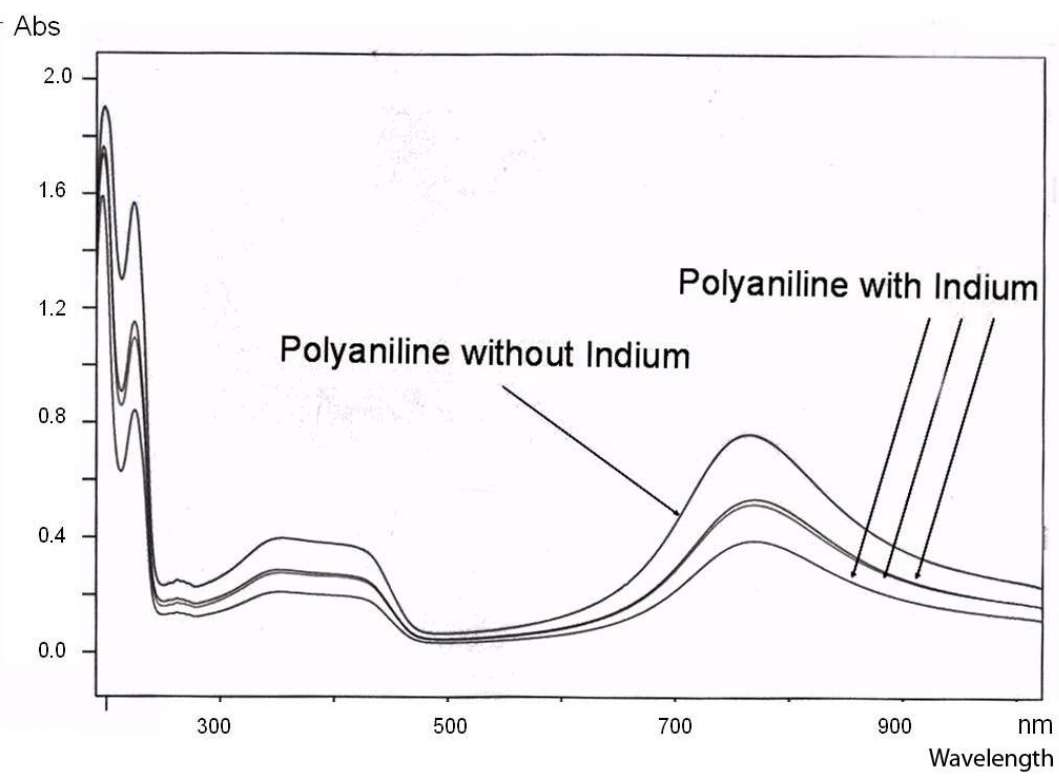

The general feature of all spectra is that the absorption strength around $800 \mathrm{~nm}$ decreases with proceeding complex formation, also the absorption strength around $450 \mathrm{~nm}$ decreases, with an isosbestic point appearing around $390 \mathrm{~nm}$, and the absorption in the UV region increases. These effects are the most impressive in the formation of the polyaniline-Cu complex. This is not so surprising as the amount of $\mathrm{Cu}^{1+}$ in this complex is 1 per about 12 aniline units, while in the case of the other ions $\left(\mathrm{Fe}^{2+}\right.$, $\mathrm{Zn}^{2+}, \mathrm{In}^{2+}$ ) the relation is roughly $1: 24$, as was found by elemental analysis.

These spectra clearly show that new compositions have been formed, true polyaniline/metal ion complexes.

The conclusion that we have drawn (in the case of $\mathrm{Cu}$ ) with $\mathrm{Cu}^{1+}$ comes from two different experiments: In printed circuit board finishing with the immersion tin process ORMECON CSN, where a water dispersion of polyaniline is used as a $\mathrm{Cu}$ surface preparation pretreatment called "predip" (trade name OMP 7000), the Cu complex plays a key role. Here, in contrast to other 
immersion tin processes not using such an organic metal predip, the relation between $\mathrm{Cu}$ release and Sn deposition is almost exactly 2:1 [21] which corresponds to a net reaction equation such as:

$$
2 \mathrm{Cu}+\mathrm{Sn}^{2+} \rightarrow \mathrm{Sn}+2 \mathrm{Cu}^{1+}
$$

For competitive tin deposition processes, a relation of roughly 1.5 to 1.0:1 has been observed which indicates a mixture of $\mathrm{Cu}^{2+}$ and $\mathrm{Cu}^{1+}$ or, in extreme cases only, $\mathrm{Cu}^{2+}$ to be released.

In addition, x-ray photoelectron spectroscopy (XPS) spectra of the $\mathrm{Cu}$ after treatment with the organic metal (in comparison to untreated $\mathrm{Cu}$ ) clearly show the $\mathrm{Cu}^{1+}$ oxidation stage [1] (p. 535).

The conclusion that in the case of iron the Fe(II) oxidation state is involved, has been drawn based on earlier work in connection with studies of the reaction mechanism leading to the passivation of iron and steel and its subsequent enhanced corrosion protection which was found to proceed via the $\mathrm{Fe}^{2+}$ ion [22].

For the polyaniline/indium complex, we additionally performed cyclovoltammetric studies [23]. While the pure polyaniline shows two oxidation and, correspondingly, two reduction peaks, the polyaniline-In complex only shows one single oxidation peak (at a significantly higher voltage) and one corresponding reduction peak (Figure 5).

Figure 5. Cyclovoltammogram of Polyaniline dispersions with and without indium. The figure shows the 5th cyclovoltammogram (CV) scan on PAni layers (PAni with and without In, respectively) deposited from dispersion onto Indium/Tin oxide (ITO); CVs performed in propylenecarbonate/0.1 $\mathrm{M} \mathrm{TEABF}_{4}$ at $25 \mathrm{mV}$.

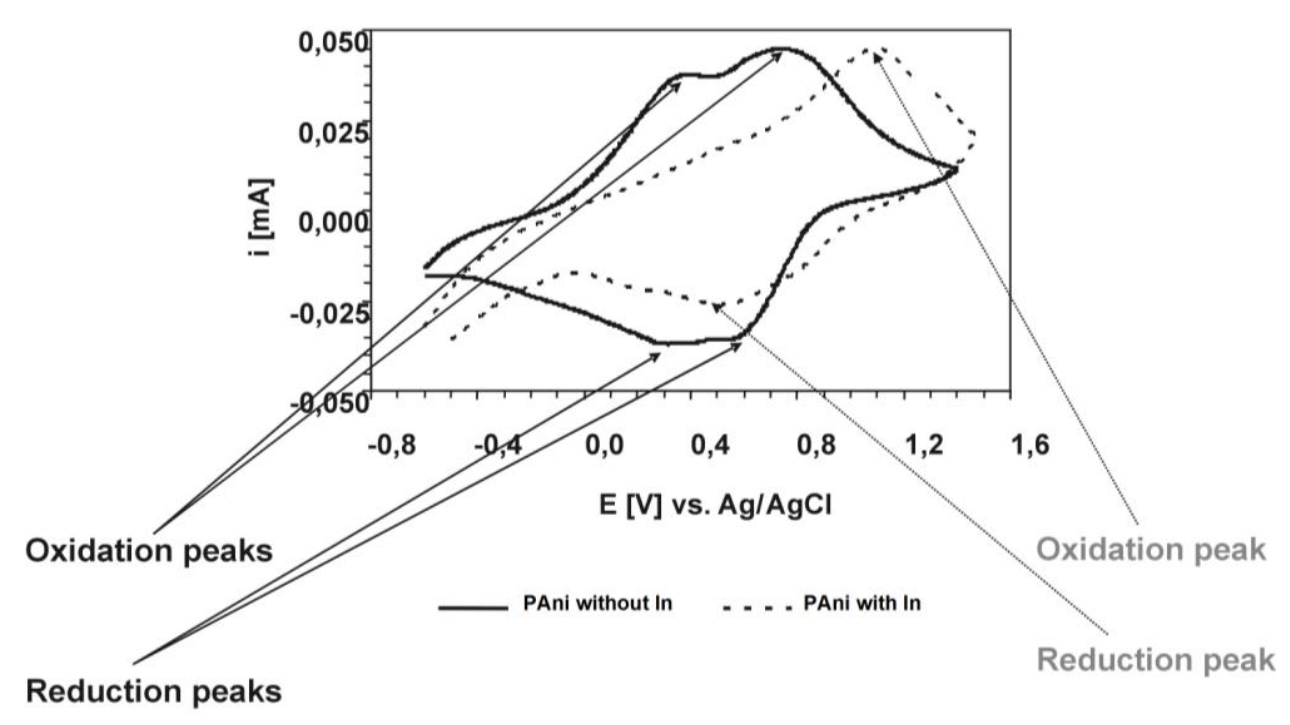

To conclude, it can be stated that polyaniline forms metal ion complexes via a reaction with the base metal, however with a low coordination number $\left(1: 12\right.$ in the case of $\mathrm{Cu}^{1+}, 1: 24$ in the case of $\left.\mathrm{Me}^{2+}\right)$. The exact structure of these complexes is not known. Three of these complexes are already commercially used: The $\mathrm{Cu}$ complex in printed circuit board finishing; the Fe complex in corrosion protection; and the In complex in hole injection layers for organic light emitting diodes (OLEDs). 


\subsection{X-Ray Diffraction Studies}

For additional insight, we performed $\mathrm{x}$-ray diffraction studies, but this time down to the range of $2 \theta=2^{\circ}$. For this study, we used polyaniline taken from different stages of the multi-step dispersion process. Figure 6 shows spectra of the raw (dry) polyaniline powder taken directly after polymerization and samples from different stages of the first dispersion step (in total, for commercial products, three subsequent dispersion steps are used [24]). The starting conductivity (raw powder) is $5 \mathrm{~S} / \mathrm{cm}$, the maximum conductivity after the first dispersion step is $30 \mathrm{~S} / \mathrm{cm}$. Figure 7 shows spectra of two samples taken from films which have been deposited after the third dispersion step.

As can be seen in Figures 6 and 7, the originally dominant peak around $2 \theta=25^{\circ}$ is weakening (= lower degree of crystallinity) and shifted to higher angle values (= denser packing, as previously observed and commented on in the introduction section), while the peak around $2 \theta=20^{\circ}$ slightly increases in intensity and shifts to lower angle values.

With increasing conductivity, especially detectable in Figure 7 , a peak at around $2 \theta=3.5^{\circ}$ (originally very weak) emerges and becomes the most prominent one. This $2 \theta$ value represents a diffraction plane distance of around $2.2 \mathrm{~nm}$, hence smaller than the $3.5 \mathrm{~nm}$ subunits found in the SAXS experiments. This means that within the $3.5 \mathrm{~nm}$ subunit, some order is emerging which is characterized by a diffraction plane pattern with $2.2 \mathrm{~nm}$ distance.

Figure 6. X-ray diffraction of polyaniline starting with the raw powder (red curve), following the progress of dispersion in the first dispersion step (from purple over green to blue curve, the latter representing the final stage of the first dispersion step); the black curve represents the same material as the blue curve after extracting all dispersion additives.

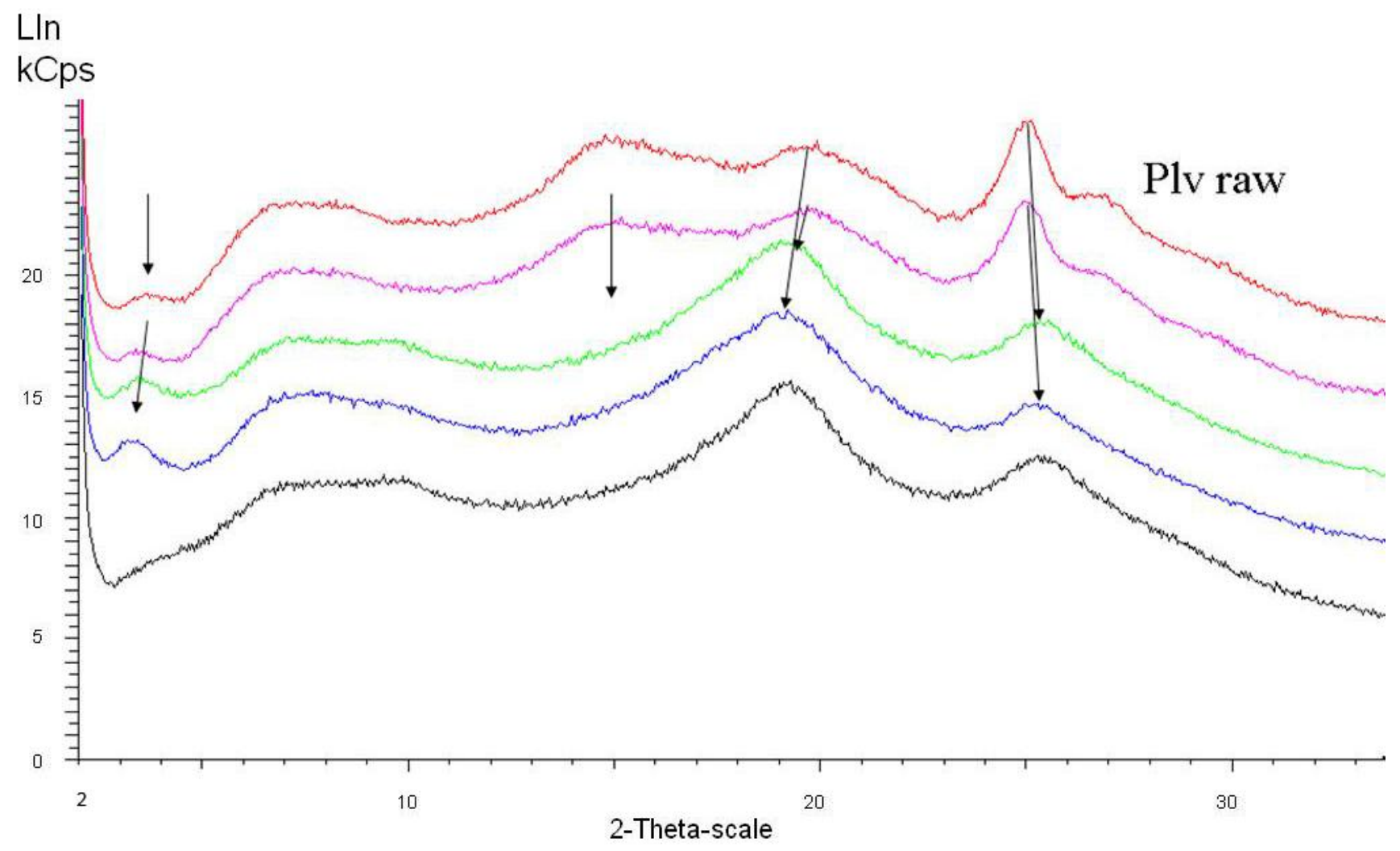


Figure 7. X-ray diffraction of polyaniline films deposited after completed dispersion steps.

The blue curve represents a film having $50 \mathrm{~S} / \mathrm{cm}$, the red curve $100 \mathrm{~S} / \mathrm{cm}$ conductivity.

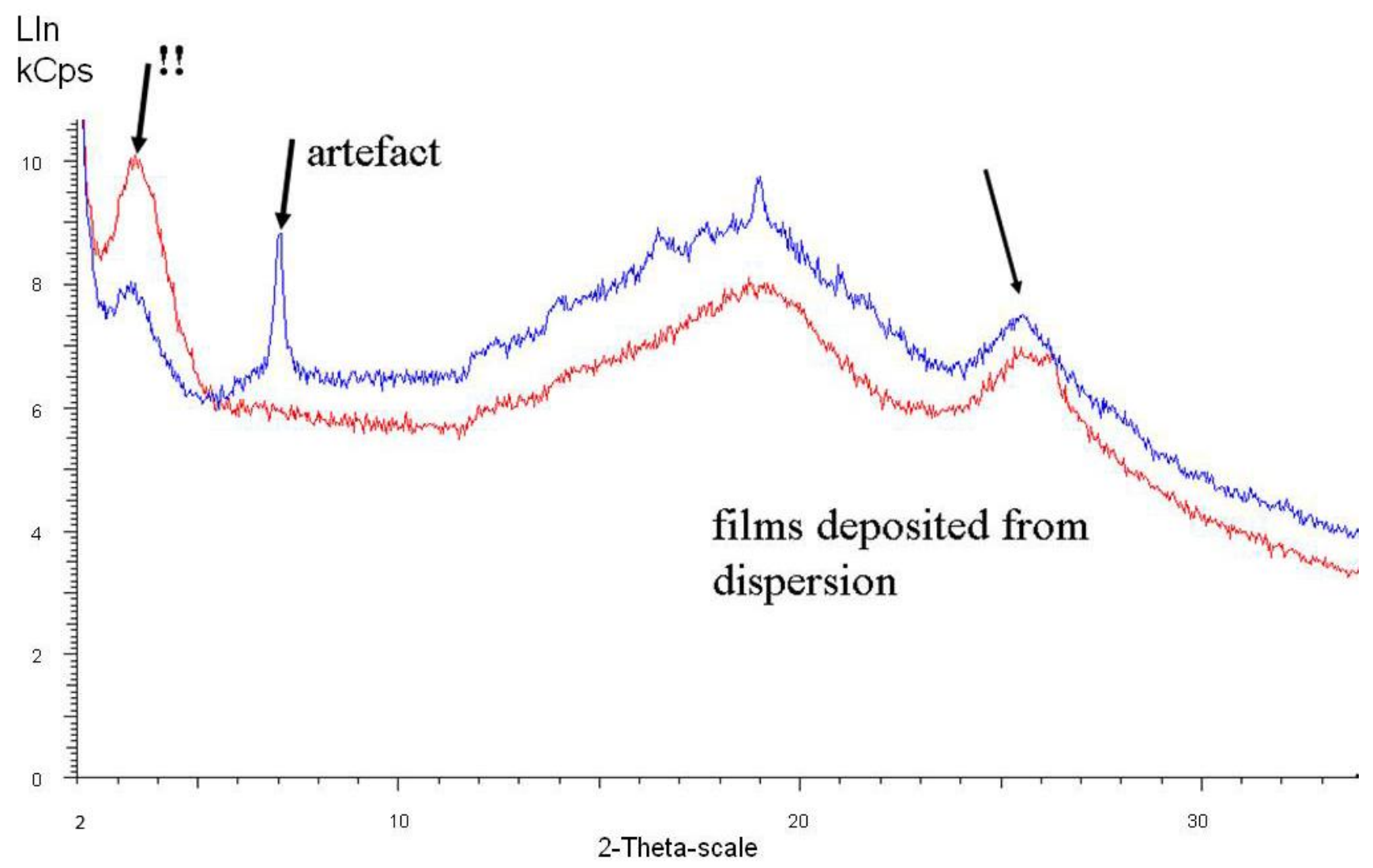

\subsection{A New Structure Model for the Organic Metal Polyaniline}

Before developing the new structure model, a few more facts needed to be considered. First, some older results should be mentioned concerning the estimated molecular weight. From MALDITOFF experiments, we know that polyaniline (at least the one we are synthesizing) exhibits a chain length of maximal 12 aniline units [17]. Second, some interesting results published by G. Wallace and coworkers a few years ago helped us make the next step towards a structure model [25]: Polyaniline can be synthesized in optically active form when using optically active dopants which is not so surprising; but what is very surprising is that it retains its optical activity after removal of the optically active dopants, although the neutral form, the so-called "Emeraldine Base", no longer has asymmetric carbon atoms. However, it can be optically active if the (relatively short, see above comment about molecular weight) chain elements are not straight, but bent into a helical form.

It should also be noted that the strength of the optical activity is very high, which is indicated by the circular dichroism effect being one order of magnitude larger than that of the optically active dopants used. This means that the optical activity induced by the helical structure of the chains is very strong.

Table 1 shows a summary and an attempt to interpret the observed peaks and their changes with increasing conductivity. 
Table 1. Summary of peaks and their changes in x-ray diffraction.

\begin{tabular}{|c|c|c|c|}
\hline \multicolumn{3}{|c|}{ Peak at $2 \theta$ (and distance in $\AA$ ) } & Interpretation \\
\hline $27^{\circ}$ & & vanishing, merging & $\operatorname{Ar} \leftrightarrow \operatorname{Ar}($ different distance) \\
\hline $25^{\circ}$ & 3.6 & merging with $27^{\circ}$ & $\operatorname{Ar} \leftrightarrow \operatorname{Ar}$ (finally only one distance range) \\
\hline $20^{\circ} \rightarrow 19^{\circ}$ & $4.4 \rightarrow 4.7$ & shifting & density fractions homogenized, more fractions with 1.6 and $1.8 \mathrm{~g} / \mathrm{cm}^{3}$ \\
\hline $15^{\circ}$ & & vanishing & \\
\hline $7-10^{\circ}$ & $9-15$ & broad & thickness of polyaniline chain including dopant \\
\hline $4^{\circ} \rightarrow 3.5^{\circ}$ & $22 \rightarrow 25$ & & helix turn distance \\
\hline
\end{tabular}

We need to add a final observation: While the primary particles of polyaniline (raw polymer powder) are more or less globular in shape (cf. STM pictures as shown in [26]), the form of the primary particles after all dispersion steps are completed and films exhibiting a conductivity of up to $600 \mathrm{~S} / \mathrm{cm}$ can be deposited, are not globular at all any more, rather ellipsoidal in form, see Figure 8 .

Figure 8. STM picture of highly conductive organic metal, deposited from dispersion onto highly oriented pyrolytic graphite (HOPG).
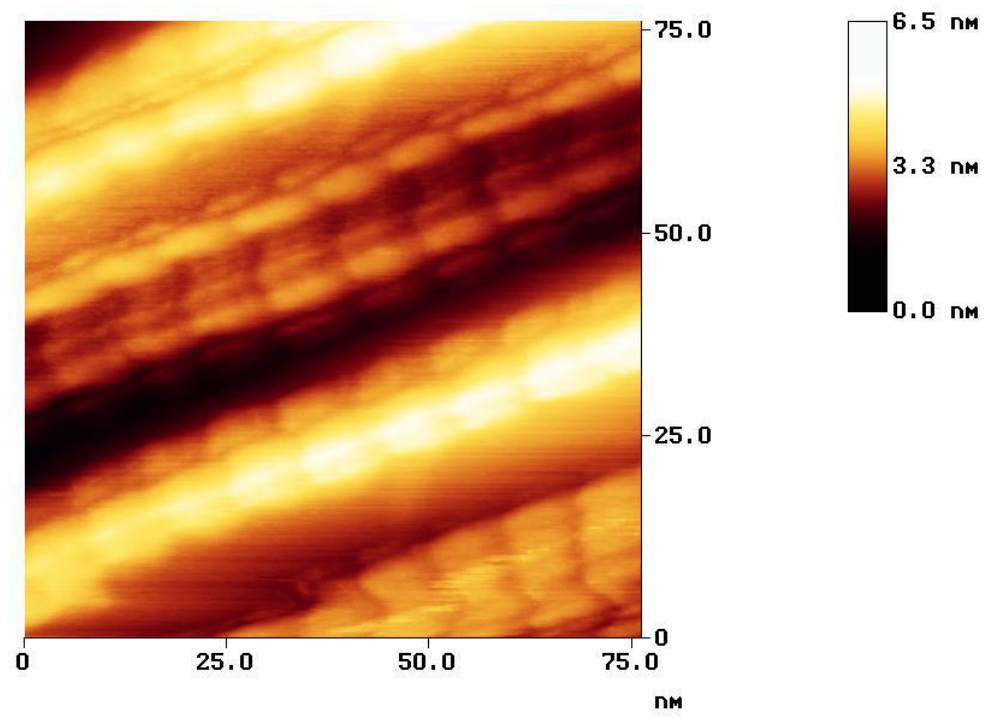

We are now ready to draft the new structure model and a model for understanding: (a) why dispersion is capable of increasing the conductivity and transforming the insulator type of conductor to a metallic type; (b) what structure features are responsible for higher conductivity.

The new model consists of the following elements:

- primary particle size of around $10 \mathrm{~nm}$ (globular form) before dispersion

- after completed dispersion, the form is ellipsoidal with a dimension of 13 (long diameter) and $6 \mathrm{~nm}$ (short diameter)

- the primary particle contains a certain number of polyaniline molecules which attain an average diameter of roughly $3.5 \mathrm{~nm}$

- the chain thickness (including dopants) is about $1.2 \mathrm{~nm}$

- the chain is coiled forming a (short) helix 
- before dispersion takes place, the short helical polyaniline chains are almost randomly oriented within the mainly globular primary particle

- upon the strong force induced by the dispersion steps, the particles not only become denser, but also the helical chains are orienting (at least partially) allowing correlation between the helix turn distance $(2.2 \mathrm{~nm}$ ) of each of the now oriented helices (see Figures 9 and 10)

- the arrangement of the short helices after dispersion is (in optimal or ideal case) such that effectively a longer helix is formed (see Figure 10)

- $\quad$ it is tempting to hypothesize that in the case of the $\mathrm{Cu}$ complex, one helix can complex one $\mathrm{Cu}$ ion, and in case of the $\mathrm{Fe}$ (and other $\mathrm{Me}^{2+}$ complexes) two helices would complex one ion

- we do not know where the dopants are located - inside the helix, "on top", or "at the bottom" of the helical chain? ("top" and "bottom" referring to the helix direction)

Figure 9. Structure model for raw undispersed polyaniline.

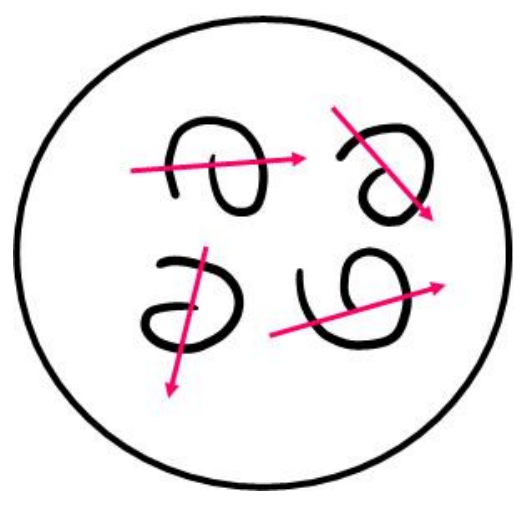

Figure 10. Structure model for the primary particle after completed dispersion.

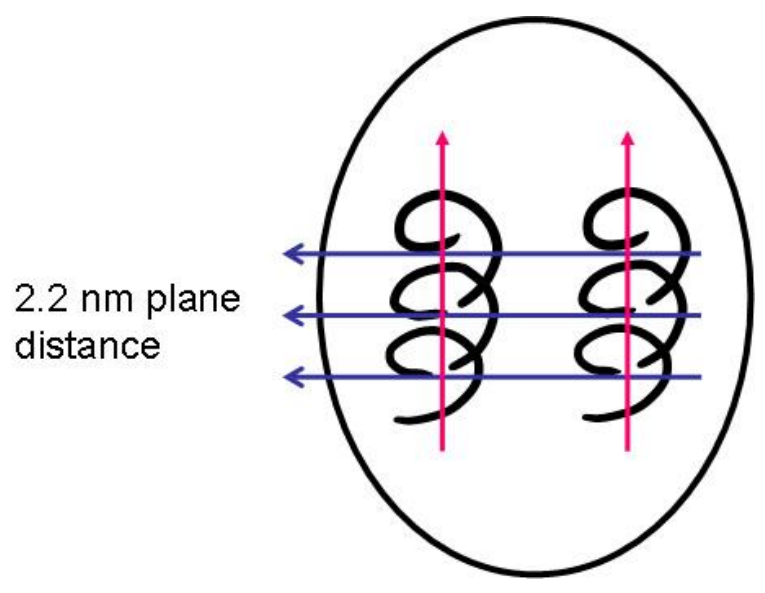

\section{Experimental Section}

Preparation of samples: polyaniline polymerization was carried out using the parameters described in [27] with the modifications described in [28]. The dispersion and sample preparation was carried out as described in [31].

UV-Vis spectra of diluted dispersions were recorded by an UV-Vis spectrophotometer Specord S100 from Analytik Jena. 
Cyclic voltammograms were recorded in a three-electrode electrochemical cell with a potentiostat/galvanostat PGSTAT 30 from Autolab. The OC test specimen served as working electrodes; a platinum wire as counter electrode; and a silver/silver chloride (in $3 \mathrm{M} \mathrm{KCl}$ ) reference electrode was used.

X-ray diffractograms have been made at Avecia, Inc. [29]

Scanning tunneling microscopy was performed at University of Muenster [31] and [30].

\section{Conclusions}

It was possible to develop a new structure model for the organic metal polyaniline. This model fits all of the available information related to the structure of polyaniline. Generally spoken, it describes polyaniline as a material built from relatively short helically formed chains which assemble within primary particles. These particles, about $10 \mathrm{~nm}$ in size, are globular for the raw polymer, and the helices are relatively disordered. During dispersion, the conductivity increases and the conductive polymer becomes an organic metal: The helices are more ordered, building some more elongated helical structures, and the primary particle changes to be ellipsoidal.

\section{Acknowledgements}

Such long-term research would not be possible without many collaborators (first within Zipperling Kessler, then within Ormecon) to whom I am very grateful. I would especially like to mention Joerg Posdorfer, Holger Merkle, Melanie Rischka, Susanne Gleeson and Marco Thun who contributed to basic research as well. Many more people have contributed to application development in printed circuit board finishing, corrosion protection, OLEDs, electroluminescence, ITO replacement and others.

\section{References and Notes}

1. Wessling, B. Conductive Polymers as Organic Nanometals. In Handbook of Nanostructured Materials and Nanotechnology; Nalwa, H.S., Ed.; Academic Press: New York, NY, USA, 2000; Volume 5, pp. 501-575.

2. Wessling, B. Dispersion as the Key to Processing Conductive Polymers. In Handbook of Conducting Polymers, 2nd ed.; Skotheim, T., Elsenbaumer, R., Reynolds, J., Eds.; Marcel Dekker, Inc.: New York, NY, USA, 1998, pp. 467-530.

3. Wessling, B. Dispersion Hypothesis and Non-Equilibrium Thermodynamics: Key Elements for a Materials Science of Conductive Polymers. Synth. Met. 1991, 45, 119-149.

4. Wessling, B. Critical Shear Rate-the Instability Reason for the Creation of Dissipative Structures in Polymers. Z. Phys. Chem. 1995, 191, 119-135.

5. Srinivasan, D.; Rangarajan, G.; Mietzner, T.; Lennartz, W.; Wessling, B. Dispersion Induced Insulator-to-Metal Transition in Polyaniline. Eur. Phys. J. 2000, E2, 207-210.

6. Wang, Z.; Li, C.; Scher, E.; MacDiarmid, A.; Epstein, A. The Granular Metal Concept for Polyaniline. Phys. Rev. Lett. 1991, 66, 1745-1748.

7. Marquardt, P.; Nimtz, G.; Mühlschlegel, P. On the Quasi-Static Conductivity of Sub-Micrometer Crystals. Solid State Commun. 1988, 65, 539-542. 
8. Nimtz, G.; Marquardt, P.; Gleiter, H. Size-Induced Metal-Insulator Transition in Metals and Semiconductors. J. Cryst. Growth 1986, 86, 66.

9. Nimtz, G.; Enders, A.; Marquardt, P.; Pelster, R.; Wessling, B. Size-Limited Conductivity in Sub-Micrometre Metal Particles. Similarities with Conducting Polymers? Synth. Met. 1991, 45, 197-201.

10. Pelster, R.; Nimtz, G.; Wessling, B. Fully Protonated Polyaniline: Hopping Transport on a Mesoscopic Scale. Phys. Rev. B 1994, 49, 12718-12723.

11. Nimtz, G.; Stahlhofen, A. Universal Tunneling Time for All Fields. Ann. Phys. (Berlin) 2008, 17, 374-379.

12. Wessling, B. Dispersion-The Key Tool for Understanding, Improving and Using Conductive Polymers and Organic Metals. Synth. Met. 2005, 1-3, 5-8.

13. Krinichnyi, V.I.; Roth, H.; Schrödner, M.; Wessling, B. EPR Study of Polyaniline Highly Doped by $p$-Toluenesulfonic Acid. Polymer 2006, 47, 7460-7468.

14. Epstein, A. Conducting Polymers-Electrical Conductivity. In Physical Properties of Polymers Handbook; Mark, J.E., Ed.; Springer Science and Business Media: New York, NY, USA, 2007; p. 730.

15. Lennartz, W.; Mietzner, T.; Nimtz, G.; Wessling, B. Morphological Changes in PAni-PMMA Blends during Dispersion Studied by SAXS. Synth. Met. 2001, 119, 425-426.

16. Wessling, B. Metal Complexes with Polyaniline and Process for Their Preparation. EP 1002 322, 24 November 2004.

17. Wessling, B. Polyaniline Complex with Indium, Process for its Preparation and Use. WO2007/020100, February 2007.

18. Posdorfer, J; Wessling, B. Passivation and Solderability Prevention of Copper by a Polyaniline/Silver Nanoparticle. Presentation at the International Conference on Science and Technology of Synthetic Metals (ICSM), Porto de Galinhas, Pernambuco, Brazil, August 2008; Available online: http://www.organic-nanometal.de/pdf/technology/recentpublications/ OMNanofinish_ICSM2008_final.pdf (accessed on 10 December 2010).

19. Wessling, B.; Thun, M.; Arribas-Sanchez, C.; Gleeson, S.; Posdorfer, J.; Rischka, M.; Zeysing, B. An Organic Metal/Silver Nanopoarticle Finish on Copper for Efficient Passivation and Solderability Preservation. Nano Express 2007, doi: 10.1007/s11671-007-9086-0; Available online: http://www.springerlink.com/content/63260u8428533014/fulltext.html (accessed on 10 December 2010).

20. Wessling, B. Ormecon's Organic Metal Technology-Science and Application. Presented at the International Conference on Science and Technology of Synthetic Metals (ICSM), Kyoto, Japan, 6 July 2010; paper \# 6B2-02.

21. Wessling, B. Metallic Properties due to Dispersion. In Handbook of Organic Conductive Molecules and Polymers; Nalwa, H.S., Ed.; John Wiley \& Sons: New York, NY, USA, 1997; Volume 3, pp. 497-578.

22. Wessling, B.; Schröder, S.; Gleeson, S.; Merkle, H.; Schröder, S.; Baron, F. Reaction Scheme for the Passivation of Metals by Polyaniline. Mater. Corros. 1996, 47, 439-445.

23. Posdorfer, J. Ormecon GmbH, Ammersbek, Germany. Personal communication. 
24. Wessling, B. Multistep Dispersion Process for Increasing Conductivity of Polyaniline. WO2005/070972, DE 202005010 364, 10 October 2008.

25. Pornputtkul, Y.; Kane-Maguire, L.A.P.; Innis, P.C.; Wallace, G.G. Asymmetric Proliferation with Optically Active Polyanilines. Chem. Commun. 2005, 36, 4539-4541.

26. Wessling, B.; Hiesgen, R.; Meissner, D. STM Investigations on Primary Particle Morphology of Polyaniline. Acta Polym. 1993, 44, 132-134.

27. Wessling, B. Process for the Polymerisation of Submicron Size Dispersible Particles of Conductive Polymers. EP 0700 573, 20 November 1996.

28. Wessling, B. Anisotropic Primary Particles of Conductive Polymers and Process for Their Formation.WO 2006/09/2292, DE 102005010 162, 14 June 2007.

29. Brown, B. Avecia Inc., Manchester, UK. Personal communication.

30. Chi, L. University of Muenster, Muenster, Germany. Personal communication.

(C) 2010 by the authors; licensee MDPI, Basel, Switzerland. This article is an open access article distributed under the terms and conditions of the Creative Commons Attribution license (http://creativecommons.org/licenses/by/3.0/). 